\title{
Atmospheric Media: How they Enable a Pre- reflective and Embodied Mode of Perception
}

\author{
Desiree Förster \\ University Potsdam, Department of \\ Philosophy, \\ Institute for Arts and Media \\ Am Neuen Palais 10 \\ 14469 Potsdam (Germany) \\ dfoerster@uni-potsdam.de
}

\begin{abstract}
Climate change, pollution, and scarcity of resources-the $21^{\text {st }}$ century confronts the human species with complex problems that have no phenomenological correlate in the everyday experience. Can there be a way to become sensible towards processes outside of our conscious awareness that take place in our atmospheric surrounding? This paper explores how atmospheric media (e.g., air, clouds, water) allow for an alternative embodied way of relating to our environment. What many atmospheric phenomena have in common is that they can hardly be objectified, i.e., they have no identifiable parts or clear dimensions, sometimes they are even partly or completely invisible. Despite their intangibility, we do experience their dynamic presence through our bodies, such as in humidity and other atmospheric phenomena. I argue that this mode of perception is pre-reflective and disregards the subject-object dichotomy.
\end{abstract}

Keywords: Atmosphere. Embodiment. Aesthetics. Perception. Experience. Architecture

\section{INTRODUCTION}

The $20^{\text {th }}$ and $21^{\text {st }}$ century is characterised by an increasing awareness for ecological challenges that evoke diverse disciplines of knowledge and have led to differently weighted but ongoing debates. Many motives and narrations that emerge in art and popular culture give expression to the helplessness and anxiety about the future associated with climate change. End-ofthe-world scenarios and those in which the human subject has consigned all control to inhuman powers populate our screens. On the other hand, preventive measures already become effective in "real life": architects and designers increasingly propose structural solutions that ought to protect us against extreme weather conditions, and new insurances against damage due to flooding or forest fires enter the market.

I argue that these developments express the dialectic between the perception of climate change in everyday life and its representation in the sciences. This dialectic manifests itself in a tension between the available knowledge about our current ecological situation propagated by science and a missing understanding of necessary consequences for the everyday life and our actions that would have to follow this knowledge. The main discrepancy this tension emerges from is the confrontation of processes that exceed cognitive understanding-e.g. the global and irreversible impact of an increase of the earth's surface by only 1 degree-and 
the way we experience these processes as events in our every-day: as extreme weather events, hot summers, and price fluctuations due to crop shortfalls. Even climate scientists formulate connections only cautiously between global warming and extreme weather conditions, as too many factors play a role to make clear statements.

In the following, I will analyse this dialectic in more detail and propose an alternative way to attune to processes such as climate change. This alternative brings to the foreground the bodily senses to intensify the subject's perception in a prereflective manner-a mode of perception that comes before all abstraction, representation and judgment of external objects in the mind. Thus, the prereflective attunement to processes concerns feelings and bodily sensations and operates in a first-person perspective of the consciously aware subject-and unlike the third-person perspective offered by science.

This mode of perception is not easily accessible in mundane existence; however, I argue that there are experiences that extend our awareness into this pre-reflective realm. In particular, I argue that atmospheric media (i.e., the natural elements such as air, light, etc.) enable this mode of perception because they are processual matters-these elements are constantly shifting and intangible. I will demonstrate how atmospheric media can amplify our sensitivity for processes within our own bodies as well as in our environment by presenting and discussing artworks, which explore the affordances of atmospheric media.

\section{ATTUNING TO PROCESS}

\subsection{Addressing a phenomenological problem}

Without going to deep into the debate on climate change at this point, a clarification of the difference between weather and climate is necessary to proceed. According to NASA, "the difference between weather and climate is a measure of time. Weather is what conditions of the atmosphere are over a short period of time, and climate is how the atmosphere 'behaves' over relatively long periods of time." (Nasa 2005). Climate cannot be experienced by our senses, it is a quantified representation of processes on scales that humans have no sensorial access to. Instead, what we experience in our everyday surroundings is weather.

Our lifeworld is the given experience of the relation between us and our environments that we share with other subjects (Husserl 1954/1970). Especially in industrialized civilizations, the role weather plays in this lifeworld stands in contrast to the complex challenges on the planetary level as they are partly represented in climate predictions. This contrast has been considered a key issue, implicating a phenomenological problem (Rudiak-Gould 2013). Planetary processes such as warming oceans and the increase of global surface temperature are mediated in form of scientific data and not experienced in a first-person perspective. Even though climate change is a re-occurring topic in the news, the consequences of these global changes on a local scale are still abstract for most people in industrialized countries-whereas developing countries feel its impact more strongly.

This is in part due to how contemporary urban infrastructure balances changes in the atmosphere and regulates the environment to create a stable "indoor climate" in people's homes. For example, temperature and humidity are regulated in 
a way to maintain a standardized comfort level for humans (ISO 7730 2005).

This introduces a dialectic between the growing urgency to respond to seemingly available knowledge-to start acting on it-and the personally perceived lack of concern. To resolve this contradiction, I see two necessary ways to proceed: First, the boundary between the third-person perspective of the Natural Sciences and the first-person perspective that makes up our every-day experience and sense of self must be overcome. While Natural Sciences are able to represent complex processes at various scales through data, studies in Arts and Humanities explore alternative ways of mediating these processes and their impact on human experience. An alliance of these fields that allows to maintain the differences in methodologies and concepts could create learning environments that promote new perceptual experiences and ways of relating to our atmospheric surroundings. Second, we need a new theory of media that accounts for the environmental dimension of our perception as well as the human body's sensitivity prior to rational thought.

\subsection{Mediating processes}

Our every-day experiences cannot account for the complexity of our lifeworld. What we experience is increasingly structured by computational networks. These networks that embed us into our (climatic) environments become more and more opaque in their ways of mediation. While for example meteorological technologies and satellites offer information about climatic processes that exceed our direct access, they also introduce a referential gap between the acting subject and its environment. In the wake of computational media, the phenomenological subject seems to be lost, replaced by interrelated processes on different scales:
Our immediate interactions with weather are extended temporally and spatially through global atmospheric sensors at both the micro scale of surface-based meteorological instruments and the macro scale of the satellite. We shift easily between distance and proximity and between human and machinic modes of perception. (Randerson et al. 2015:17)

Computational processes occur at extremely accelerated time scales, typically well below the thresholds that would allow for a human perceptual experience. The end product of these processes is increasingly opaque since their mode of operation is inhuman (Galloway \& Thacker 2007). These processes of mediation themselves, which shape the environment we inhabit, its affordances and thereby our very becoming, are not part of our experience. The direct connections to our physical being in the world and the relations we form through our actions remain in the background.

The dimension that gets lost in this operation of computational media is in fact the dimension that fostered the concept of the human subject as a higher-order cognitive being. Media scholar Mark B. Hansen identifies this as the main feature of what he calls twenty-first-century media (Hansen 2015). Thereby the concept of the human subject and the role of its cognitive capacities get into a precarious situation. The loss of human-centred experience leads to a potential increase of "worldly experience" (Hansen 2015), which is not centred around a subjective experience but is environmental in character. According to Hansen, this domain of a new worldly sensitivity does not equate subjective experience with lived experience any longer.

To summarize my point of departure, the dialectic we face with climate change today challenges our understanding of experience in radically decoupling experience from knowledge. The global scale of planetary processes such as climate change as well as the local 
computational processes that regulate the micro-climatic conditions of our every-day, happen on temporally and spatial scales outside of our perceptual capacity.

In the following I want to suggest an alternative perspective on how we relate to our environment to tackle this problematic. This perspective assumes that our corporeality grants a special access, which does not lose its vigour, but on the contrary, must be intensified. As embodied beings, we are sensitive on scales outside of higher order cognition and therefore able to attune to processes not graspable by our rational-thinking mind alone. For this, I will first examine the affordances of atmospheric media.

\section{ATMOSPHERIC MEDIA}

The term atmosphere goes back to the Greek atmos (vapour, steam) and sphaira (sphere) and refers to the layer of gas that surrounds the earth. The later and rather metaphorical use to describe moods and feelings is today so deeply engrained in our language and culture (e.g., as political atmosphere, the atmosphere of a place, etc.) that one could argue it is no longer a metaphor either.

The range of how we experience an atmosphere in the latter sense is wide, going from an "atmosphere that comes over us" to one that "is not noticeable at all" (Schmitz 2014:69). Following this observation, Hermann Schmitz attributed atmospheres the status of a "quasiobject"-as such it does neither belong to the category of the subject nor the object but expands and interconnects both (Schmitz 2014:75).

\subsection{Atmospheres as experience in the arts}

This quasi-object value of an atmosphere, alongside with its evasiveness makes it a powerful concept and even material for the arts. Considering atmospheres not only as processes themselves but also as media that can bring processes to experience, makes them interesting for a reconsideration of a theory of media.

Artworks that use atmospheres as media tend to not revolve around a solid object at its centre but instead engage the audience in atmospheric processes, which challenge the audience in a particular way; may it be a gust of wind blowing lightly through a room such as in Ryan Gander's The Invisible Pull (Gander 2012), or the clouds in Fujiko Nakaya's Cloud Parking that envelop visitors on the roof top of a city parking lot (Nakaya 2012), or the heavy rain that never wets the visitors in Random International's Rain Room (Random International 2012). The atmospheres in these artworks are not approached in their immaterial quality to induce a certain mood, but in their material dimension that is also subject to climate science: the very elements atmospheres are composed of. Thereby, these atmospheres become a medium of the experience themselves.

\subsection{Aesthetics of Atmospheres}

The corporeal sensations of the participants are centre stage in these atmospheric artworks and thus processes that are normally not perceived are intensified. The focus is shifted from the contemplation of an art object to the way the sensual body is sensitive to experiences internal and external to it. As the Gernot Böhme states:

\footnotetext{
The aesthetics of atmosphere shifts attention from the 'what' something represents, to the 'how' something is present. In this way, sensory perception as opposed to judgment is rehabilitated in aesthetics and the term 'aesthetic' is restored to its original meaning, namely the theory of perception. (Böhme 1993: 114)
}

As the body is able to sense changes in the atmosphere, such as a sudden breeze or shift in temperature, an intensification of such changes can become an aesthetic experience, in which the change discloses the ways the body is embedded in its environment. This is Böhme's "how" we 
experience that something is present. Böhme goes further and states that atmospheres are the primary object of perception. Neither being feelings nor forms, figures, or objects, nor their constellations, atmospheres are perceived as the background that serves for the formation and abstraction of objects, forms, and colours against this background (Böhme 1993:122).

This interplay of foreground and background in perception is a necessary condition for perception to arise (MerleauPonty 1945/2005). For example, the brightness of a surrounding is experienced as the background against which the colour of an object appears:

\begin{abstract}
Lighting and reflection, then, play their part only if they remain in the background as discreet intermediaries, and lead our gaze instead of arresting it. (...) The shade does not become really a shade (...) until it has ceased to be in front of us as something to be seen, but surrounds us, becoming our environment in which we establish ourselves. (Merleau-Ponty, 1945/2005:362)
\end{abstract}

The background that enables the foregrounding of things in allowing contrasts and meaning to appear, gives way to the constant formation of feelings, abstractions, subjectivities.

Atmospheric media have the potential to heighten our sensitivity to processes in our surrounding without ever offering a distinct object of perception. In fact, the fascination with atmospheric media might exactly lie in their evasiveness. They are neither subjective nor objective but seem to mark the in-between of subjects and objectsnot as a zone of differentiation but as moving beyond the bodily boundaries, interrelating processes internal and external to it.

The two understandings of what an atmosphere is that we covered, i.e., an elemental-based atmosphere (e.g., the planet's atmosphere) as well as the felt atmosphere (e.g., a certain tune or mood) cannot be identified as objects or subjects due to their processual character.

An aesthetic of atmospheres therefore differs from intellectualism in aesthetics, which assumes that knowledge about an object can be derived from its appearance (Böhme 1995). If it is not the rational subject that is able to experience atmospheres, how then can the subject of atmospheric media be described in more detail?

\section{ARTISTIC EXAMPLES OF AN AESTHETICS OF ATMOSPHERES}

In the following I will briefly introduce two artworks that apply atmospheric media in spatial installations. I will describe how, in these artworks, the body of the perceiver extends to the environment through the atmospheric media. Furthermore, I will discuss how the bodily sensitivity and the sensible environment come to the foreground as mediators for processes in lived experience (Merleau-Ponty 1945/ 2005:78).

\subsection{Artwork \#1: Hormonorium}

The installation Hormonorium by JeanGilles Décosterd \& Philippe Rahm, which is depicted in figure 1 , emanates climatic conditions of $3000 \mathrm{~m}$ altitude. To achieve this, the floor is covered with transparent panels under which 528 fluorescent tubes are placed, which emit a white light that reproduces the solar spectrum (with UV-A and UV-B). The room's temperature controlled to be around $16^{\circ} \mathrm{C}$ and the oxygen levels is kept relatively low. 


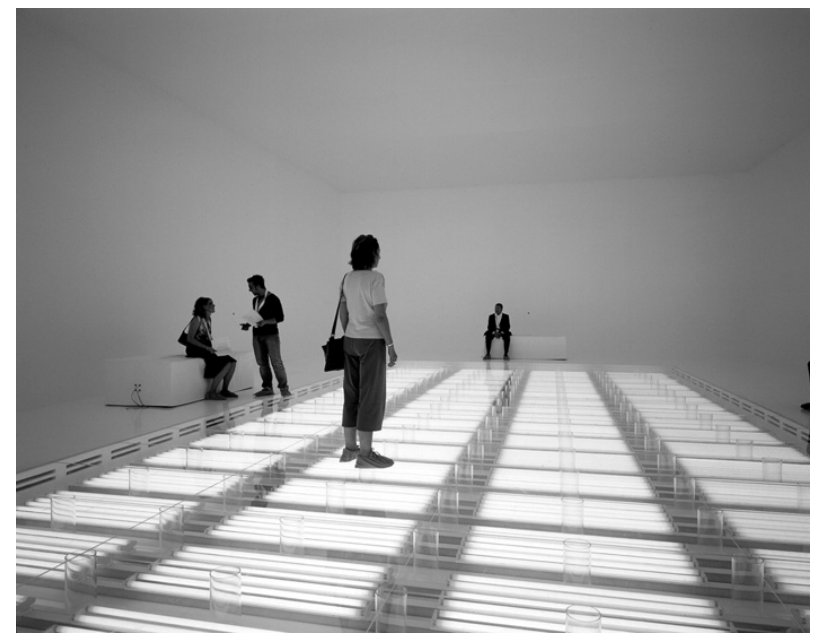

Figure 1: Décosterd \& Rahm, Hormonorium, $8 e$ architecture biennale of Venice, 2002 Photo: Jean-Michel Landecy.

Altitude sickness can be experienced already around $2000 \mathrm{~m}$, symptoms can appear after a short time under these conditions (Gale Encyclopedia of Medicine 2008).

Thus, depending on the duration of the visitor's stay in the installation, the low oxygen levels might induce a disorientation, headache, or even unreasonable behaviour. The light rays can lead to the feeling of being more awake, up to euphoria and agitation. In integrating biological principles in spatial arrangements as explicit co-creators, qualities like response, adaptation, and processes such as decay and disturbance, become sensual. In focusing on the transformation and exchange of energy and matter, rather than fixed relations or objects, a processual way of being-in the world is captured. The Hormonorium challenges our understanding of Homeostasis. Homeostasis, from Greek as in same or steady, refers to processes in organisms that actively maintain stable conditions required for survival. In the installation, the materiality of homeostatic processes can be experienced. In putting the body's sensitivity towards homeostatic processes inside and outside at centre stage, the body not only mediates spatial experience, it becomes the space itself. This attuning to homeostatic processes comes with the experience of change from one experiential state to another; it differs from the common understanding of affect as something that overcomes us abruptly from an external origin.

To summarize, conscious experience of the biochemical processes in Hormonorium arises through the attribution of importance to bodily processes that are intensified by the change in the atmosphere, recognition of previous experiences, information gathered about the installation, and other visitors. Here, the atmosphere becomes something outside of the experiencer and at the same time it is felt inside, experienced and mediated by the subjective physical feelings. Intensifying the experience of the thermoregulatory capacity of bodies allows then to mediate the interrelation between bodies and environments as transitional in character. Experience then is neither just physical nor just conscious and abstract, but instead oscillates in between.

\subsection{Interoception as attunement to internal processes of the body}

In this first artwork, the use of atmospheric media allows to experience homeostasis as process-not as a stable equilibrium-, which then becomes a central structural element of the aesthetic milieu. The body of the visitor can mediate these processes, because it not only allows sensory experience external to it in the environment but also internal: it channels the sensory experience in the mode of interoception-the sensory apparatus that perceives internal processes, which we sometimes refer to as gut feeling. The body as medium for atmospheric and biochemical processes discloses the interrelatedness of organism and environment. This interrelatedness is in fact not a stable state but processual itself and pre-reflective and conscious experience emerge as part of phases of these processes and are not qualities of distinct domains (Simondon 1967/1992). 
Ultimately, this artwork is made of the visitor's experience itself, as they experience shifts between different states on the biochemical as well as emotional level. Another way to frame this is to see that the atmospheric elements are not made accessible to our senses as objects of perception, but instead they are experienced as the process of sensing itself: the atmosphere of the Hormonorium is not outside of the visitors, it envelops them into the space, leads to the production of hormones and affords new action potential.

For one of the artists, Rahm, architecture must be understood as the design of atmospheres that create "a pocket of space" (Rahm 2013) within the void, that is not immaterial but instead rich with elements and processes that have effects on our bodies. Understanding and using these effects does not only potentially lead to the design of spaces that bring into accordance the chemical and physical properties of our environments with the chemical and physical processes of our bodies, but can also sensitize to the invisible, unrepresentable, that makes up the majority of our actual life-world. If the invisible material that flows in our atmosphere is shown today to have deep impacts on our life-world-may it be in form of emissions that impact our future climate or garbage that pollutes our oceans-a higher sensitivity for these metabolic flows is necessary. But how can we account for responsible and ethical behavior if the very resource of our knowledge is defined by is elusiveness, constantly transforming and hard to objectify?

\subsection{Artwork \#2: Urban Algae Canopy}

In the previous example, oxygen played a central role as the element that embeds the audience into space. If we consider oxygen as part of the infrastructure as well as the biochemical processes that allow bodies to engage with our environment, the distinction between the body and environment becomes obsolete.

The installation Urban Algae Canopy by EcoLogicStudio demonstrates this relationship by using oxygen as a mediator between humans and algae. The piece, depicted in figure 2, is a transparent structure in which each facet is filled with a fluid containing microalgae. The on-going respiration of visitors, which exhale $\mathrm{C02}$, adds to the algae's nutrient base. Thus, the growth of the algae is influenced by the number of visitors. If the number of algae in a particular facet increases, the facet becomes more and more opaque, dynamically creating a shade, as well as the release of more oxygen into the canopy. Accompanying the changes in shading and air quality, the visitor's movement is tracked by motion sensors and influences the rate at which the algae fluid is moved through the facet, by means of hydraulic pumps, as well as additional nutrition that is automatically adjusted.

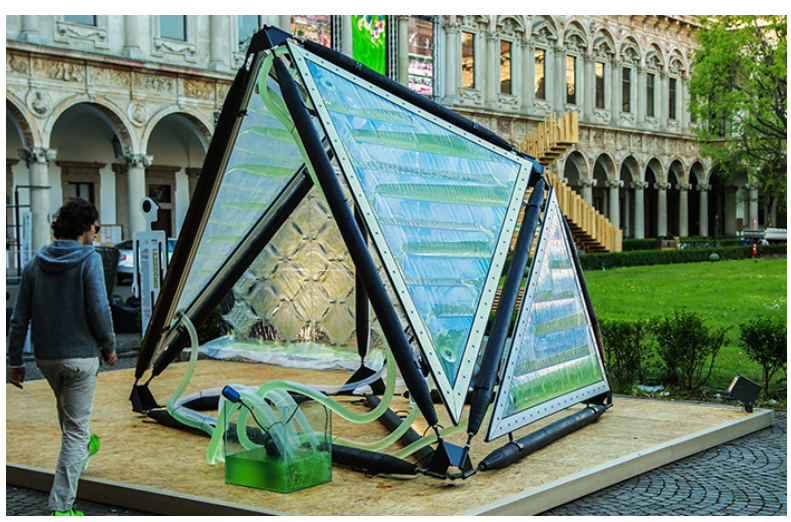

Figure 2: Urban Algae Canopy, by EcoLogicStudio.

In this artwork, the on-going biochemical exchange between humans and algae becomes part of the environment itself. It is not so much the individual visitor's interaction that becomes experiential as it is the previous visitor's behaviour that effects the environment in a way that it becomes part of the interaction that follows. A feeling of sharing an environment is induced, through experiencing the effects of others on one's own experience of space. 
Environments that respond to different scales, such as macro-scale (weather patterns) and social scales (the behaviour of organisms) can make us aware of the spatiality and presence of others in experiencing the effects of our interrelations with them.

\subsection{Stigmergy as attunement to processes in the external surrounding}

The ability to notice and communicate what appears as meaningful for our being in the world, the development and exercise of concern, begins with sensitivity for homeostatic processes. As we learn to interpret relations between the homeostatic state of our body and our environment, we establish habits and concerns that ensure the stability of this relation, e.g. we dress according to seasons, invest in heating and air condition, and so on. While this comes natural to us, babies must rely on their care-taker's interpretation of their voicing whenever the homeostatic stability of their bodies is misbalanced. Some researchers from the cognitive science argued that one of the main purposes of early social interactions actually is the regulation of the infant's homeostasis (Fotopoulou \& Tsakiris 2017). Therefore, embodied interactions with others "allow the developing organism to mentalize its homeostasis and hence they constitute the core of our embodied subjectivity" (Fotopoulou \& Tsakiris 2017:6).

As I have covered, the pre-reflective sensitivity to our surroundings does not rely on representations created by a cognitive mind, and can therefore also be observed in other, non-human animals. In the context of Urban Algae Canopy, the concept of stigmergy comes to mind. This concept, first used by entomologist PierrePaul Grassé, described a mechanism of coordination used by insects, especially those that exhibit self-organization in large groups such as how ants leave pheromone trails to demarcate food sources. As such, stigmergy grew as a concept that now entails cooperation and self-organization in large groups, in which the agents communicate through changes in the shared environment. In Urban Algae Canopy it is precisely the shared environment that carries traces from the previous visitor's movements, much like the stigmergy observed in ant colonies. The traces are expressed by the living infrastructure, by the way the algae grow and enrich the air with oxygen.

In this way, the interrelatedness of human beings and their surroundings expands in time; it becomes part of the sensual experience of sharing the space with others (be it other human visitors or microscopic organisms). Therefore, the development towards an aesthetic that makes these processes and their subsequent connections outside of our conscious awareness perceivable, may function as a platform to approach a new subjectivity, a being-in and -of the world as a part of atmospheric environments.

\section{CONCLUSION}

The artworks that I discussed here demonstrate how perception can be restructured, when atmospheric media become part of the aesthetic milieu. The chosen artworks (Hormonorium and Urban Algae Canopy) are installations that utilize elemental forms such as air or water to express the processual character of the relations formed between organisms and environments.

What is idiosyncratic about atmospheric phenomena is that they cannot be reduced to a mere representation of data (e.g., as happens in science communication of phenomena such as climate change). Atmospheres can only be perceived through bodily engagement with the space, in a mode of perception that is prereflective. The perceiver feels the unfolding of matter in space (e.g., wind, etc.), and the impacts on feelings and 
sensations that arise from it (e.g., the impact of oxygen levels on our behaviour) as well as in the immediate spatial surrounding (e.g., the algae growth in response to carbon dioxide in the air).

Atmospheric media are able to shift the attention of the audience to the 'how' something is experienced by the sensitive body and the way feelings and intentions arise from these experiences. I argued that this is enabled by the behaviour of these element-based atmospheres: On the microscopical level, air, water, light, are in constant transformation. Therefore, they can become a medium for the experience of processes. This experience of processes is primarily corporeal as I have demonstrated with the example of these two artworks, as the subject itself becomes the medium of the art pieces: the corporeal subject as the locus of continues processes of adjustment. Therefore, the effects of atmospheric media cannot be separated from our lived experience or from our lifeworld.

Lastly, I argued that an aesthetics of atmospheres is able to conciliate and connect scientific findings with the sensitive life world; this aesthetic can make distinct scaling of space and time palpable and recognizable for a bodily sensitive subject.

\section{REFERENCES}

\section{BOOK}

Böhme, G. (1995) Atmosphäre. Frankfurt am Main: Suhrkamp.

Frost, S. (2016) Biocultural Creatures: Toward a New Theory of the Human. Durham: Duke University Press.

Galloway, A.R., Thacker, E. (2007) The exploit: a theory of networks, Electronic mediations. Minneapolis: University of Minnesota Press.
Hansen, M. B. N. (2015) Feed-Forward: On the Future of Twenty-First-Century Media. Chicago, London: University of Chicago Press.

Husserl, E. (1970, orig. 1954). The Crisis of European Sciences and Transcendental Phenomenology. Evanston: Northwestern University Press.

Merleau-Ponty, M. (2005, orig. 1945) Phenomenology of Perception. Translated by Colin Smith. London: Routledge.

Schmitz, H. (2014) Atmosphären. Freiburg: Karl Alber.

\section{BOOK CHAPTER}

Simondon, G. (1992, orig. 1967) The Genesis of the Individual. In: Jonathan Crary \& Sanford Kwinter (ed(s)). Incorporations. New York: Zone Books. 297-319.

\section{JOURNAL ARTICLE}

Fotopoulou, A. \& Tsakiris, M. (2017) Mentalizing homeostasis: The social origins of interoceptive inference. Neuropsychoanalysis, 19 (1). 3-28.

Böhme, G. (1993) Atmosphere as the Fundamental Concept of a New Aesthetics. Thesis Eleven, 36. 113-126.

Grassé, P. P. (1959) La reconstruction du nid et les coordinations interindividuelles chez Bellicositermes natalensis et Cubitermes sp. la théorie de la stigmergie: Essai d'interprétation du comportement des termites constructeurs. Insectes Sociaux 6 (1). 41-80.

Rudiak-Gould, P. (2013) We have seen it with our own eyes. Why we disagree on climate change visibility. Weather Climate Society 5. 120-132. 
Randerson, J. et al. (2015) Weather as Medium: Art and Meteorological Science. Leonardo 48 (1). 16-24.

\section{TECHNICAL DOCUMENTS}

ISO 7730 (2005) Ergonomics of the thermal environment -- Analytical determination and interpretation of thermal comfort using calculation of the PMV and $P P D$ indices and local thermal comfort criteria. Geneva: International Organization for Standardization.

\section{INTERNET SOURCE}

Gale Encyclopedia of Medicine (2008)

Altitude sickness. Medical Dictionary. Available from: https://medicaldictionary.thefreedictionary.com/altitude+si ckness [01.08.2018].

Nasa (2005) What's the Difference Between Weather and Climate? Nasa.

Available from:

https://www.nasa.gov/mission_pages/noaa -n/climate/climate_weather.html

[15.06. 2018].

Décosterd, J. G. \& Rahm, P. (2002) Hormonorium. Available from:

http://www.philipperahm.com/data/projects /hormonorium/ [15.06. 2018].

Ecologic Studio (2014) Urban Algae Canopy. Available from:

http://www.ecologicstudio.com/v2/project.p hp? idcat $=7 \&$ idsubcat $=59$ \&idproj= 129

[15.06. 2018].

Nakaya, F. (2012) Cloud parking. a as architecture. Available from:

http://aasarchitecture.com/2013/04/cloudparking-by-fujiko-nakaya.html

[15.06. 2018].

Random International (2013) Rain Room. The Museum of Modern Art. Available from: https://www.moma.org/calendar/exhibition s/1352 [15.06. 2018].

Gander, R. (2012) I Need Some Meaning I Can Memorize (The Invisible Pull). Frieze. Available from:

https://frieze.com/article/ryan-gander-0 [15.06. 2018].

Rahm, P. (2013) Open House Lecture: Philippe Rahm "Constructed Atmospheres". Harvard GSD. Available from:

https://www.youtube.com/watch?v=NP6EB TwGcug [15.06. 2018]. 